\title{
OCIO DEPORTIVO EN JÓVENES POTENCIALMENTE VULNERABLES: BENEFICIOS PERCIBIDOS Y ORGANIZACIÓN DE LA PRÁCTICA
}

\author{
SPORTS LEISURE IN POTENTIALLY VULNERABLE YOUNG PEOPLE: \\ PERCEIVED BENEFITS AND ORGANIZATION OF PRACTICE

\section{JUVENTUDE DESPORTOS DE LAZER POTENCIALMENTE VULNERÁVEIS: PERCEBIDA BENEFÍCIOS E ORGANIZAÇÃO DE PRÁTICA}

\author{
Raúl FRAGUELA VALE*, Ángel DE-JUANAS OLIVA**, \\ Ricardo FRANCO LIMA*** \\ *Universidade da Coruña, ${ }^{* *}$ UNED, ${ }^{* * *}$ Instituto Politecnico de Viana do Castelo
}

PALABRAS CLAVE: juventud deportes tiempo de ocio actividades físicas socialización
Fecha de recepción del artículo: 27.VII.2017 Fecha de revisión del artículo: 18.1X.2017 Fecha de aceptación final: 26.1X.2017

\begin{tabular}{l|l} 
PALABRAS CLAVE: & $\begin{array}{l}\text { RESUMEN: La adquisición de un ocio físico deportivo habitual es uno de los objetivos que } \\
\text { juventud } \\
\text { deportes } \\
\text { tiempo de ocio } \\
\text { actividades físicas } \\
\text { socialización }\end{array}$ \\
$\begin{array}{l}\text { da y mejoren su calidad de vida. A su vez, la práctica de ocio deportivo es reconocida como } \\
\text { una herramienta de primer orden para lograr la socialización de los jóvenes potencialmente } \\
\text { vulnerables. Este estudio analizó la incidencia de la vulnerabilidad en los hábitos de ocio de- } \\
\text { portivos de los jóvenes; en sus preferencias de práctica físico deportiva de forma estructu- } \\
\text { rada o libre; } y \text {, finalmente, en la percepción sobre los beneficios que conlleva esta práctica. } \\
\text { Para ello se realizó un diseño de investigación cuantitativo en el que se aplicó un cuestionario } \\
\text { elaborado ad hoc a un total de } 2694 \text { estudiantes españoles de Educación Post-obligatoria no }\end{array}$ \\
\hline
\end{tabular}

CONTACTAR CON LOS AUTORES: Raúl Fraguela Vale. Universidad de A Coruña. Facultad de Ciencias de la Educación. Campus de Elviña, s/n. 15071 A Coruña. Telf.: 881014619. E-mail: raul.fraguela@udc.es.

FINANCIACIÓN: El presente trabajo es resultado de los proyectos de investigación: “De los tiempos educativos a los tiempos sociales: la construcción cotidiana de la condición juvenil en una sociedad de redes. Problemáticas específicas y alternativas pedagógico-sociales" (Proyecto Coordinado EDU 2012-39080-C07-00) y de los subproyectos: "De los tiempos educativos a los tiempos sociales: la construcción cotidiana de la condición juvenil en una sociedad de redes: Problemáticas específicas y alternativas pedagógico-" (EDU 2012-39080-C07-01) y "De los tiempos educativos a los tiempos sociales: Ocio, formación y empleo de los jóvenes en dificultad social" (EDU 2012-39080-C07-07), cofinanciados en el marco del plan nacional l+D+i con cargo a una ayuda del Ministerio de Economía y Competitividad, y por el Fondo Europeo de Desarrollo Regional (FEDER, 2007-2013). “Educar el ocio: realidades y perspectivas en clave intergeneracional, integral e Inclusiva en una sociedad de redes" (Proyecto EDU2015-65638-C6-1-R), financiado en el marco del plan nacional I+D+i con cargo a una ayuda del Ministerio de Economía y Competitividad. 
universitaria, de los que 785 fueron considerados como participantes en potencial riesgo de vulnerabilidad. Los resultados revelaron que existen diferencias entre los jóvenes potencialmente vulnerables y no vulnerables en cuanto a la práctica de actividades de ocio deportivo. La población en riesgo de vulnerabilidad manifiestó hábitos de ocio menos activos que los no vulnerables. Asimismo, se encontró que los jóvenes vulnerables tenían una mayor tendencia a la práctica de ocio deportivo por su cuenta, frente a los no vulnerables que preferían realizar deporte de forma organizada. De la misma forma, los jóvenes potencialmente vulnerables percibieron menores beneficios de la práctica deportiva que los no vulnerables. De los resultados se deriva la necesidad de aplicar actuaciones socioeducativas estructuradas para promocionar la práctica de ocio deportivo entre los jóvenes más vulnerables.

KEY WORDS:

youth

sports

leisure time

physical activities

socialization

ABSTRACT: The acquisition of a frequent sports leisure practice is one of the objectives that our society must achieve in order to ensure proper health and an improvement of life quality of young people. In turn, sport leisure practices are considered a frontline tool to foster socialization of potentially vulnerable young people. This study examined the effect of vulnerability in sports leisure habits in young people; in their preferences of physical and sporting practices through an organized or autonomous way; and finally, in the perceptions of the benefits that these practices entail. For this purpose, a quantitative research design was conducted, administering an ad hoc questionnaire to a sample of 2694 post-obligatory and non-university students in Spain; 785 of them were labelled as students at potential risk of vulnerability. The results revealed the existing differences between potentially vulnerable and non-vulnerable young people regarding sports leisure practices. The population at risk of vulnerability displayed less active leisure habits than the rest. Likewise, it was found that vulnerable youth had a greater tendency to practice sports leisure activities in an autonomous way, unlike non-vulnerable young individuals, who preferred an organized practice. Similarly, potentially vulnerable young people obtained fewer benefits from sports leisure activities than non-vulnerable ones. The results proved the need to implement structured socio-educational interventions in order to promote sports leisure practices among the most vulnerable young people.

PALAVRAS-CHAVE: juventude desporto tempo de lazer atividades físicas socialização
RESUMO: A aquisição de hábitos de lazer na atividade física e desportiva é um dos objetivos da nossa sociedade para que os nossos jovens tenham uma saúde adequada e uma melhor qualidade de vida. Por sua vez, a prática desportiva como lazer é reconhecida como uma ferramenta fundamental para desenvolver a socialização dos jovens potencialmente vulneráveis. Este estudo pretende analizar a incidencia da vulnerabilidade nos hábitos de lazer desportivo dos jovens; na sua preferencia na realização das atividades físicas e desportivas de forma estruturada e livre; e, finalmente, na perceção sobre os beneficios que advém da prática desportiva. Para isso, realizou-se um desenho de investigação quantitativo em que se aplicou um questionário elaborado ad hoc a um total de 2694 estudantes espanhois de Educação Pós-obrigatória não universitária. Da totalidade dos participantes, 785 foram considerados como potenciais risco de vulnerabilidade. Os resultados revelam que existem diferenças entre os jovens potencialmente vulneráveis e não vulneráveis no que respeita à prática de atividades de lazer desportivo. Evidencia-se que os participantes de risco vulnerável manifestam hábitos de lazer desportivo menos átivo do que os não vulneráveis. Ainda, os jovens vulneráveis têm maior tendência para a prática de lazer desportivo, enquanto os não vulneráveis preferem realizar atividades desportivas de forma organizada. Da mesma forma, os jovens vulneráveis percebem menores beneficios da prática desportiva quando comparados aos não vulneráveis. Os resultados obtidos apresentam a necessidade de aplicar estratégias socioeducativas estruturadas para promover a prática de desporto como lazer entre os jovens mais vulneráveis.

\section{Introducción}

En la sociedad actual, el estilo de vida de las personas está íntimamente relacionado con la utilización de su tiempo de ocio. El disfrute de este tiempo es un derecho básico e individual que ha de facilitarse y promoverse entre todos los ciudadanos (Cuenca, 2014). Entre los más jóvenes está proliferando, cada vez más, un tipo de ocio sedentario relacionado con la utilización de dispositivos tecnológicos. El auge de los sistemas y tecnologías de la comunicación como modos de entretenimiento está ganando terreno a otras formas de ocio entre las nuevas generaciones constituidas por nativos digitales (Melendro, García-Castilla, \& Goig, 2016; Valdemoros, Sanz, \& Ponce de León, 2017). Este cambio acelerado hacia estilos de vida sedentarios sitúa los niveles de práctica de actividad física diaria de la población española por debajo de las recomendaciones de la Organización Mundial de la Salud (Ministerio de Sanidad, Servicios Sociales e lgualdad, 2014) y constituye uno de los grandes factores de riesgo íntimamente vinculado al incremento de la obesidad, la diabetes, la hipertensión y otras enfermedades no transmisibles (Sotomayor, Aquino, Jiménez, \& Trejo, 2014).

Frente al ocio pasivo, la práctica de ocio deportivo mantiene un poder de atracción considerable 
entre los jóvenes de nuestra sociedad. No hay que olvidar que el deporte trasciende a la población juvenil como espectáculo social por influencia de los medios de comunicación (Gómez, Puig, \& Maza, 2009). Los medios contribuyen a enfatizar modelos deportivos basados en el alto rendimiento que, a su vez, sirven de principal reclamo para muchos jóvenes que deciden iniciar sus primeros pasos en la práctica de una actividad deportiva.

La práctica de ocio deportivo se ha popularizado por sus beneficios asociados a la promoción y mantenimiento de la salud corporal pero, en los últimos tiempos, se ha incrementado de manera relevante la práctica de actividad físico deportiva relacionada con la mejora de la imagen corporal, especialmente entre las chicas (Olive, Byrne, Cunningham, \& Telford, 2012; Valdemoros, Sanz, \& Ponce de León, 2012). Esta preocupación por la imagen corporal se destaca como uno de los principales motivos para practicar actividad físico deportiva junto con la satisfacción vital per se y la diversión vinculada con este tipo de ocio (Zullig, \& White, 2011; Pedisic, Greblo, Phongsaban, Milton, \& Bauman, 2015; Ahedo, \& Macua, 2016; Fraguela, Varela, \& Sanz, 2016).

El ocio deportivo presenta otros beneficios menos visibles para los jóvenes, pero especialmente relevantes desde una perpectiva social. Así, el deporte posee un gran potencial integrador, promueve la comunicación e interacción entre personas de diferentes nacionalidades y culturas (Consejo Superior de Deportes, 2010) y facilita los procesos de socialización en contextos juveniles (Gutiérrez del Pozo, 2011). La actividad deportiva tiene una amplia tradición como recurso para la prevención de conductas antisociales, favorece la asunción de responsablidades, la toma de decisiones y la resiliencia (Hellison, 2011). Por todo ello, la práctica de ocio deportivo entre los jóvenes potencialmente vulnerables es considerada como herramienta de desarrollo e integración en diferentes contextos sociales (Spaaij, 2009; Haudenhuyse, 2013; Fernández-García, Poza-Vilches, \& Fiorucci, 2015; López-Noguero, Sarrate, \& Lebrero, 2016). Así, Martínez (2016) subrayó la importancia de los hábitos deportivos en jóvenes vulnerables asociándolos con el cuidado de la salud, la socialización y la contención afectivo-social. En la misma línea, Chalip y Hutchinson (2016) destacaron la necesidad de plantear actividades deportivas enfocadas al desarrollo de habilidades sociales en esta población.

A pesar de los trabajos revisados, se encuentran pocos estudios que contrasten las prácticas de ocio deportivo de los jóvenes potencialmente vulnerables con la de aquellos que no lo son. Si bien, los datos de la Encuesta sobre los hábitos deportivos en España 2010 advierten que, a pesar de la tendencia de los últimos años que evidencia que el ocio deportivo de la población juvenil general ha experimentado un aumento en nuestra sociedad, se detecta una disminución relevante entre aquellas personas que no tienen estudios y manifiestan un estatus socioeconómico bajo. Así mismo, si se atiende a la organización de la práctica deportiva, parece existir un incremento generalizado de la práctica no estructurada entre los jóvenes de entre 15 y 24 años. La modalidad de ocio deportivo (libre u organizado) es un factor a tener en cuenta, ya que repercute en la adherencia a la actividad físico deportiva y en los beneficios de la misma entre nuestros jóvenes (Ahedo, \& Macua, 2016). Así, durante la juventud, la práctica de actividades de ocio deportivo dirigidas potencia las relaciones sociales y contribuye a consolidar hábitos deportivos en la adultez (Ransdell, Vener, \& Sell, 2004), mientras que la autogestión de las actividades de ocio deportivo mejora la motivación intríseca y la satisfacción de los jóvenes (Lazcano-Quintana, \& Caballo-Villar, 2016).

\section{Justificación y objetivos}

Este trabajo responde a resultados parciales de una gran investigación en red sobre el tiempo de ocio de los jóvenes. Dentro del ocio en la juventud, la dimensión social de las actividades físicas deportivas va más allá de la promoción de sentimientos de pertenencia y participación. El deporte es una herramienta que contribuye a desarrollar la cohesión e inclusión social de jóvenes vulnerables (Comisión Europea, 2011). Por ello, resulta necesario considerar el estudio de los hábitos de ocio deportivo de los jóvenes potencialmente vulnerables e indagar sobre las diferencias que existen en relación con los jóvenes no vulnerables. Para el presente trabajo, se concretan tres objetivos. El primero, describir los hábitos de ocio deportivo de los jóvenes en riesgo de vulnerabilidad y de los que no son vulnerables. El segundo, analizar las preferencias de los jóvenes potencialmente vulnerables y no vulnerables a la hora de realizar actividades físico deportivas de ocio de forma organizada o de forma libre. Finalmente, el tercero, examinar si la percepción de los beneficios del ocio físico deportivo de los jóvenes vulnerables sigue un patrón similar a la de sus compañeros no vulnerables.

\section{Metodología}

La investigación ha seguido un proceso metodológico de tipo cuantitativo dentro del marco de un proyecto coordinado en red realizado por siete 
universidades españolas. El diseño de este estudio transversal ha sido descriptivo, inferencial y ex post facto. Para este trabajo, se han explorado las elecciones de ocio deportivo de los jóvenes potencialmente vulnerables y no vulnerables. Asimismo, a partir de los objetivos mencionados en el epígrafe anterior, se desprenden las dos hipótesis que guían este estudio, las cuales se presentan a continuación. La primera es que la variable vulnerabilidad marca una diferencia en la preferencia de realizar deporte de forma organizada. La segunda es que la variable vulnerabilidad puede influir en la percepción de los beneficios de la práctica de ocio físico deportivo.

\subsection{Participantes}

Para la selección de los participantes se realizó un muestreo probabilístico estratificado mediante afijación proporcional, tomando como referencia territorial las áreas Nielsen y, dentro de éstas, la titularidad de centro, el sexo y curso. En total, participaron 2694 estudiantes de Educación Post-obligatoria no universitaria. La participación fue elevada, quedando garantizada la representatividad de la muestra con un error del $1.9 \%$ y un nivel de confianza del $95 \%$.

La Tabla 1 recoge los principales datos de identificación de los participantes, atendiendo a sus características socio-descriptivas:

\begin{tabular}{|c|c|c|c|c|}
\hline \multicolumn{5}{|c|}{ Tabla 1: Datos de identificación de la muestra } \\
\hline Edad $\bar{x}(D T)$ & Sexo (\%) & Vulnerabilidad (\%) & Titularidad Centro (\%) & Zonas' (\%) \\
\hline $19.15(1.57)$ & $\begin{array}{l}\text { Chico (51.3) } \\
\text { Chica }(48.7)\end{array}$ & $\begin{array}{l}\text { Si (29.1) } \\
\text { No (70.9) }\end{array}$ & $\begin{array}{l}\text { Pública (78.6) } \\
\text { Privada laica (4.5) } \\
\text { Privada religiosa (16.9) }\end{array}$ & $\begin{array}{l}\text { Noreste (17.6) } \\
\text { Levante (12.8) } \\
\text { Sur (28.4) } \\
\text { Centro (22) } \\
\text { Noroeste (11.2) } \\
\text { Norte (8) }\end{array}$ \\
\hline
\end{tabular}

\subsection{Instrumentos}

Se elaboró un cuestionario ad hoc sobre la organización de los tiempos académicos y de ocio. En este artículo se muestran los resultados correspondientes a las siguientes variables, pertenecientes a los bloques sobre descripción del alumnado, tiempo libre y salud y calidad de vida del cuestionario:

Vulnerabilidad: para el estudio de la juventud vulnerable se construyó una variable según los siguientes criterios:

- Estudios: se incluyó a todo el alumnado de Programas de Cualificación Profesional Inicial (PCPI) y de Formación Profesional Básica (FPB).

- Ingresos familiares: se seleccionó al alumnado perteneciente a aquellas unidades familiares con ingresos iguales o inferiores a quinientos euros.

- Situación familiar: se seleccionaron las situaciones en las que el alumnado no tiene madre ni padre, se encuentraba en acogimiento o pertenecía a la categoría "otros" (por no encajar en ninguno de los tipos familiares más habituales).

- Situación profesional del padre y de la madre: se incluyeron los casos en los que los dos progenitores estaban desempleados o que, al menos uno, estaba en el paro y el otro se dedicaba a tareas domésticas. También se incorporaron las situaciones en las que el alumnado solo tenía madre o padre si éste/a estaba desempleado/a o realizaba tareas del hogar.

- Nivel de estudios del padre y de la madre: se incluyeron los casos en los que ninguno de los progenitores tenía estudios terminados (o la persona con que vivía con el alumno/a en el caso de tener solo padre o madre).

- Nota media: se incorporó a todo el alumnado con una nota media inferior a 5 .

- Satisfacción familiar: se incluyeron todos aquellos casos que estaban poco o nada satisfechos con su vida familiar (valores 1 o 2 sobre un valor máximo de 5).

Una vez seleccionados los participantes que cumplían alguno de los criterios fijados, se obtuvo una población en potencial riesgo de vulnerabilidad de 785 (29.1\% del total de la muestra).

Ocio deportivo (entre las 3 actividades de ocio más importantes): los participantes tuvieron que indicar la actividad de ocio más importante que realizaban de un listado de 49 posibilidades. Estas actividades se presentaron agrupadas en 9 categorías: televisión y radio, ocio digital, deporte y actividad física, turismo y excursionismo, actividades culturales, fiestas/celebraciones, juegos 
(que no sean de ordenador), asociacionismo y voluntariado y otras actividades. A partir de los resultados de estas tres variables se construye la variable ocio deportivo, con las categorías: ninguno, una actividad, dos actividades y tres actividades (en función del número de veces que aparece una actividad de deporte y actividad física entre las tres actividades de ocio más importantes).

Beneficios percibidos: los jóvenes indicaron los principales beneficios que percibían al practicar las actividades de ocio más importantes, a saber: mejora de la condición física, diversión, aprendizaje-creación, desarrollo de habilidades y socialización. A través de una escala tipo likert de 5 puntos valoraron el grado de acuerdo con la existencia de estos 5 beneficios. En los casos en los que la juventud realizaba más de una actividad de ocio deportivo, se calculó la media aritmética para cada beneficio. Por ejemplo, para el beneficio de mejorar la condición física, si un sujeto valoró una de sus actividades de ocio con un 4 y otra con un 2, su experiencia global de ocio deportivo respecto a este beneficio fue valorada con un 3 .

Organización de la práctica: se indicó si realizaron las actividades de forma libre (por su cuenta), de forma organizada o de ambas formas. En el caso de realizar más de una actividad de ocio deportivo, se recodificó la variable en función de las modalidades organizativas de las distintas actividades. Así, por ejemplo, si un sujeto practicaba un deporte de forma libre y otro de forma organizada, la organización conjunta de su ocio deportivo fue codificada como ambos (formatos de organización).

\subsection{Procedimiento}

Dos investigadores se desplazaron a cada centro educativo para aplicar los cuestionarios en el aula. Los jóvenes participaron en la investigación de forma anónima y voluntaria, con el conocimiento y consentimiento de las familias y de los equipos directivos de los centros, siendo informados de los objetivos de la investigación. El trabajo de campo se realizó durante el curso académico 2015-2016.

\subsection{Análisis de datos}

Se realizó un análisis de tipo descriptivo e inferencial. Para la descripción de la participación en ocio deportivo, su organización y beneficios percibidos se utilizaron análisis de frecuencias y medidas de tendencia central. Para valorar el efecto del factor vulnerabilidad sobre las variables relacionadas con los beneficios del ocio deportivo se utilizó la prueba U de Mann-Whitney. Esta misma comprobación se realizó con las variables ocio deportivo y organización de la práctica a través del análisis Chi-cuadrado.

Para el tratamiento estadístico se utilizó el software informático SPSS 22.0. Al igual que en la mayoría de investigaciones con este diseño, no es posible establecer relaciones de causalidad entre las variables dependientes e independientes.

\section{Resultados}

\subsection{Ocio deportivo y vulnerabilidad}

Tal y como se indica en la Figura 1, más de la mitad de los jóvenes españoles realiza alguna actividad físico-deportiva entre sus 3 actividades de ocio más importantes. De las 9 modalidades de ocio estudiadas (electrónico, cultural, deportivo, festivo, etc.) es ocio deportivo es, con diferencia, el más practicado por la juventud. A pesar de ello, casi un $45 \%$ de los participantes no realizan ninguna actividad físico-deportiva en su tiempo de ocio.

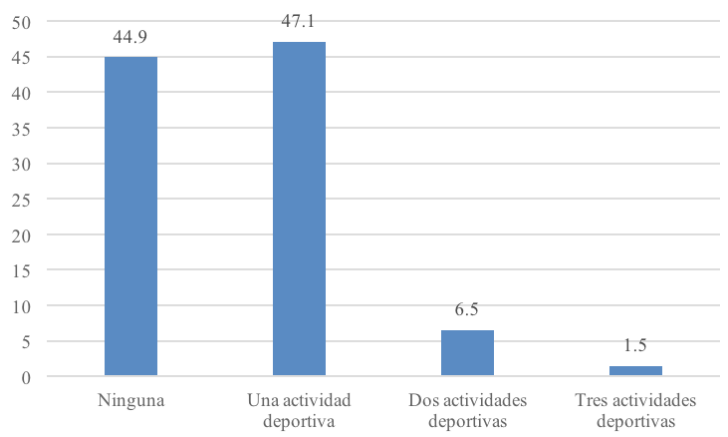

Figura 1. Ocio deportivo en jóvenes españoles

A continuación se estudia la existencia de diferencias entre los jóvenes vulnerables y no vulnerables en cuanto a la práctica de actividades de ocio deportivo. La población en riesgo de vulnerabilidad tiene unos hábitos de ocio menos activos que sus compañeros. Tienden a seleccionar en menor medida el ocio deportivo entre sus tres actividades de ocio más importantes que sus compañeros no vulnerables (Chi-cuadrado = $24.428, p<.01$ ). En la figura 2 se estudia la diferencia entre ambos colectivos en cuanto a sus preferencias de ocio. El análisis de los residuos tipificados corregidos nos indica que las diferencias significativas entre ambos grupos se encuentran en las categorías ninguna actividad deportiva y una actividad deportiva. 


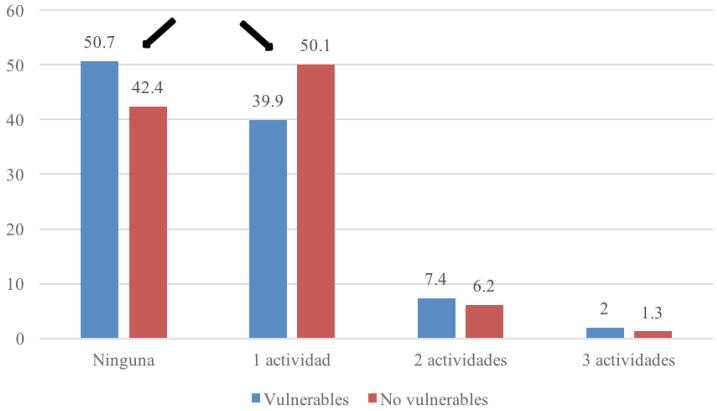

Figura 2. Actividades de ocio deportivo y vulnerabilidad

Los jóvenes vulnerables tienden a no practicar ninguna actividad deportiva durante su tiempo de ocio en mayor medida que sus compañeros, mientras que sucede lo contrario en la categoría de una actividad de ocio deportivo.

Respecto a los jóvenes con 2 y 3 actividades deportivas entre sus 3 actividades de ocio más importante, el comportamiento de vulnerables y no vulnerables es similar. Parece que el ocio deportivo funciona como referencia principal para una minoría de jóvenes -vulnerables y no vulnerables- ( $8 \%$ de la muestra y un $14.5 \%$ de los que practican alguna actividad de ocio deportivo) cuyos intereses de ocio son mayoritaria o exclusivamente deportivos.

\subsection{Organización del ocio deportivo y vulnerabilidad}

Para los siguientes análisis nos centraremos exclusivamente en la parte de la muestra que practica actividad físico-deportiva en su tiempo de ocio (55.1\%, 1486 jóvenes). Los jóvenes practican las actividades de su ocio deportivo mayoritariamente de forma organizada, en clubes, asociaciones, etc. (42.6\%). La segunda opción es practicar de forma libre $(34 \%)$ y, finalmente, un $23.4 \%$ combina ambas formas de organización. La variable vulnerabilidad marca una diferencia importante en la organización de las actividades deportivas de ocio (Chi-cuadrado = 11.135, $\mathrm{p}<$.01). Los jóvenes vulnerables tienen una mayor tendencia a la práctica deportiva por su cuenta que los no vulnerables, que prefieren claramente realizar deporte de forma organizada (Figura 3). El análisis de los residuos tipificados corregidos indica que las diferencias significativas entre los grupos se encuentran en las categorías práctica libre y práctica organizada. El comportamiento en la categoría ambas es casi idéntico en ambos colectivos.

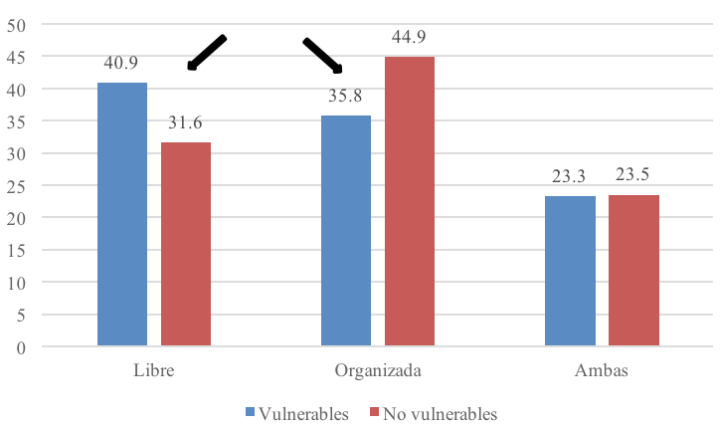

Figura 3. Organización del ocio deportivo y vulnerabilidad

El perfil de práctica deportiva de los jóvenes vulnerables tiende a ser más individual y menos estructurado que el de sus compañeros. En el apartado siguiente se estudiará si existe relación entre la vulnerabilidad y la percepción de beneficios vinculados a la práctica deportiva.

\subsection{Beneficios del ocio deportivo}

Sobre la percepción de beneficios de la práctica de un ocio deportivo, destaca la satisfacción y la consecución de una mejor forma física. La dimensión relacional también está muy presente entre los jóvenes (Tabla 2).

\begin{tabular}{|l|c|}
\hline \multicolumn{2}{|c|}{$\begin{array}{c}\text { Tabla 2: Beneficios percibidos } \\
\text { del ocio deportivo }\end{array}$} \\
\hline \multicolumn{1}{|c|}{ Beneficios (valores 1-5) } & $\overline{\mathbf{x}}(\mathrm{DT}$ ) \\
\hline Condición Física & $4.57(.74)$ \\
\hline Satisfacción & $4.65(.67)$ \\
\hline Creatividad & $3.55(1.27)$ \\
\hline Habilidades & $4.04(1.18)$ \\
\hline Socialización & $4.12(1.17)$ \\
\hline Fuente: Elaboración propia. & \\
\hline
\end{tabular}

Al comparar la percepción de beneficios de ocio deportivo de la juventud potencialmente vulnerable con la de sus compañeros, destaca que en casi todos los casos (excepto en el de la creatividad), los jóvenes potencialmente vulnerables presentan puntuaciones más bajas que los no vulnerables. En el caso de la condición física, la satisfacción y la socialización, estas diferencias son estadísticamente significativas (Tabla 3 ). 
Tabla 3: Beneficios percibidos del ocio deportivo y vulnerabilidad. U de Mann-Whitney

\begin{tabular}{|c|c|c|c|}
\hline & $\mathrm{N}$ & $\begin{array}{c}\text { Vulnerables vs No vulnerables } \\
\qquad \bar{x}(D T)\end{array}$ & Z \\
\hline Condición Física & 1330 & $4.47(.83)$ vs $4.61(.70)$ & $-2.375^{*}$ \\
\hline Satisfacción & 1317 & $4.56(.76)$ vs $4.68(.64)$ & $-2.466^{*}$ \\
\hline Creatividad & 1288 & 3.59 (1.26) vs 3.54 (1.27) & .655 \\
\hline Habilidades & 1300 & $3.99(1.17)$ vs 4.05 (1.18) & -1.081 \\
\hline Socialización & 1297 & 3.99 (1.18) vs $4.16(1.16)$ & $-2.732^{* *}$ \\
\hline $\begin{array}{l}{ }^{*} p<.05,{ }^{* *} p<.0,01 \\
\text { Fuente: Elaboración propia. }\end{array}$ & & & \\
\hline
\end{tabular}

Los jóvenes en riesgo de vulnerabilidad, por lo tanto, no solo practican deporte en menor medida que sus compañeros, sino que perciben menores beneficios de dicha práctica.

\section{Discusión y conclusiones}

En relación con nuestro primer objetivo por el que pretendíamos representar los hábitos de ocio deportivo de los jóvenes en riesgo de vulnerabilidad y de los que no son vulnerables, los resultados hallados corroboran los datos del Anuario de Estadísticas Deportivas (Ministerio de Educación, Cultura y Deporte, 2015) que reflejan que, para el intervalo de edad de 15 a 24 años, el $40 \%$ de los jóvenes más vulnerables afirman practicar una actividad física deportiva. Estos datos también coinciden los resultados del estudio de García-Castilla, De-Juanas, \& López-Noguero (2016) realizado con una muestra de similares características. Si bien, la descripción de los hábitos de ocio deportivo de los jóvenes potencialmente vulnerables también nos ha permitido evidenciar que tienden a no practicar ninguna actividad deportiva durante su tiempo de ocio en mayor medida que otros jóvenes no vulnerables. Al respecto, se confirman los resultados del Informe de UNICEF (2010) sobre el bienestar en las naciones más ricas del mundo. Según datos de este estudio, España se encuentra entre los países con mayor desigualdad en cuanto a práctica de ejercicio físico intenso. En el informe se destaca que el nivel de práctica de los estudiantes de 11, 13 y 15 años más desfavorecidos se encuentra muy por debajo de la media del país. De tal modo, "para el 'ejercicio físico intenso' de nuevo los Países Bajos tienen la menor desigualdad, seguidos de cerca por Suiza y Noruega. Los niveles más altos de desigualdad en el extremo inferior de la escala se registran en Francia, Italia y España" (UNICEF, 2010, p.15). En un mismo sentido apuntan los resultados de la Encuesta sobre hábitos deportivos en España 2010 elaborada por el Consejo Superior de Deportes. Los datos de esta encuesta indican que aquellos jóvenes de quince o más años que se encuentran sin estudios o tienen un menor estatus socioeconómico, manifiestan un interés reducido por el deporte. Esta realidad tiene implicaciones negativas en la salud del colectivo de jóvenes vulnerables, ya no solo en el presente, sino como expectativa de hábitos de ocio menos activos en otras etapas de la vida. Más allá, si se tiene en consideración que los resultados de la encuesta proponen que los antecedentes familiares de la práctica deportiva influyen en la práctica deportiva de las personas que tienen 15 años o más. De tal modo, los jóvenes se encuentran predispuestos a reproducir el modelo parental de práctica de ocio deportivo. No obstante, la incidencia de este factor ambiental y familiar en el ocio deportivo de los jóvenes más vulnerables debe ser estudiado con mayor detenimiento.

A su vez, en línea con nuestro primer objetivo, los resultados permiten considerar la existencia de un colectivo con gran afinidad por la práctica deportiva como actividad de ocio (aquellos que seleccionan dos o tres actividades deportivas entre sus tres principales preferencias de ocio) que queda fuera de esta relación entre la existencia de vulnerabilidad y la práctica deportiva. En este caso, el comportamiento de vulnerables y no vulnerables es muy similar, hasta el punto de poder ser considerados como un grupo homogéneo. De esta forma, las actuaciones socioeducativas diseñadas para promocionar la práctica de ocio deportivo entre los jóvenes vulnerables deberían centrarse específicamente en aquellos con un interés moderado por el ocio deportivo (los que 
no practican o realizan una actividad durante su ocio), ya que claramente es el colectivo con mayor riesgo de asentar ocios físicamente pasivos.

Respecto a nuestro segundo objetivo, relacionado con el análisis de las preferencias sobre el tipo de organización de ocio deportivo de los jóvenes potencialmente vulnerables y no vulnerables, en trabajos recientes se había observado un incremento generalizado para toda la población juvenil de práctica deportiva no organizada (Consejo Superior de Deportes, 2010; Ahedo, \& Macua, 2016). Esta tendencia es aún más acusada en el colectivo de jóvenes vulnerables: en nuestra investigación se identifica una menor estructuración y compromiso social por parte de estos jóvenes, al decantarse por una práctica deportiva por libre. Al respecto, coincidimos con Lazcano-Quintana y Caballo-Villar (2016), al señalar que el hecho de estar apuntado a una actividad supone un mayor grado de compromiso social y tiene efectos sobre la continuidad de la práctica. Todo ello confirma la primera hipótesis del estudio, dado que los jóvenes vulnerables no solo practican menos deporte que otros iguales, sino que aquellos que lo realizan tienden a hacerlo por libre. Este hecho marca una tendencia hacia una relación más débil con el ocio activo de este colectivo. Desde una perspectiva general, la práctica menos estructurada y sin un sentimiento de pertenencia al grupo, presenta mayor riesgo de abandono deportivo a corto-medio plazo. En cualquier caso, es necesario profundizar en los motivos por los que los jóvenes potencialmente vulnerables tienden a realizar actividades no dirigidas frente al resto de jóvenes. Resultaría especialmente interesante analizar aquellas variables contextuales que pueden estar incidiendo en la decisión de realizar deporte de manera autónoma y su impacto en esta población.

En relación a nuestro tercer objetivo, centrado en explorar la percepción de los beneficios del ocio deportivo de los jóvenes vulnerables y no vulnerables, existe una tendencia a que los jóvenes en riesgo de vulnerabilidad perciban menores beneficios en su ocio deportivo que sus compañeros, sobre todo en los tres beneficios más valorados en estas edades. Por tanto, se confirma nuestra segunda hipótesis y se manifiesta que los jóvenes potencialmente vulnerables se divierten menos, mejoran en menor medida su forma física y no se relacionan tanto con su grupo de iguales en sus experiencias de ocio deportivo. El resultado de esta percepción tiene un efecto negativo sobre la continuidad de la práctica deportiva, ya que los que perciben un menor beneficio de una actividad, tienden a abandonarla con mayor facilidad. Esto arruinaría la adherencia a la práctica deportiva de este colectivo y contribuiría a fomentar la degradación social por la que atraviesan muchos de estos jóvenes, dado que dirigirían su interés hacía otras formas de ocio menos saludables (OMS, 2013); sin olvidar que dejan de disfrutar de otros beneficios del ocio deportivo, especialmente de su potencial socializador, que promueve el desarrollo de capacidades transferibles a otros ámbitos de la vida (Ramos, Ponce de León, \& Sanz, 2010).

Por otro lado, el colectivo de vulnerables muestra un interés similar por la mejora de las habilidades mediante el deporte y por su dimensión socializadora. Los no vulnerables, aunque también están interesados en el desarrollo de sus habilidades, perciben que el deporte les permite relacionarse con los demás en mayor medida. Estos datos pueden estar informando de una relación más utilitaria que social entre los jóvenes vulnerables. Aunque hay que tomar esta interpretación con precaución, ya que las diferencias no son muy grandes, puede apuntarse una tendencia a un menor componente social en el ocio deportivo de este colectivo, lo que es coherente con su menor participación en actividades organizadas.

\section{Referencias bibliográficas}

Ahedo, R., \& Macua, A. (2016). Características de las prácticas de ocio físico-deportivas significativas de los jóvenes españoles. Revista de Psicología del Deporte, 25(2), 67-72.

Chalip, L., \& Hutchinson, R. (2016). Reinventing youth sport: formative findings from a state-level action research project. Sport in Society, Special Issue, 1-17. doi: https://doi. org/10.1080/17430437.2015.1124562

Consejo Superior de Deportes (2010). Ideal democrático y bienestar personal. Encuesta sobre los hábitos deportivos en España 2010. Recuperado de http://www.csd.gob.es/csd/estaticos/dep-soc/encuesta-habitos-deportivos2010.pdf

Cuenca, M. (2014). Aproximación al ocio valioso. Revista Brasileira de Estudos do Lazer. Belo Horizonte, 1, 21-41.

Echezarreta, R. R., Ponce de León, A., \& Sanz, E. (2010). El ocio físico-deportivo en adolescentes. La Rioja: Universidad de La Rioja.

Fernández-García, A., Poza-Vilches, F., \& Fiorucci, M. (2015). Análisis metateórico sobre el ocio de la juventud con problemas sociales. Pedagogía Social. Revista Interuniversitaria, 25, 119-141.

Fraguela, R., Varela, L., \& Sanz, E. (2016). Ocio deportivo, imagen corporal y satisfacción vital en jóvenes españoles. Revista de psicología del deporte, 25(2), 33-38. 
García-Castilla, F. J., De-Juanas, A., \& López-Noguero, F. (2016). La práctica de ocio deportivo de los jóvenes en situación de vulnerabilidad. Revista de Psicología del deporte, 25(2), 27-32.

Gómez, C., Puig, N., \& Maza, G. (2009). Deporte e integración social. Guía de intervención educativa a través del deporte. Barcelona: INDE.

Gutiérrez del Pozo, D. (2011). Psicología, educación en valores y deporte. Revista de Psicología y Educación, 7(6), 199-210.

Hellison, D. (2011). Teaching responsibility through physical activity. Champaing, IL: Human Kinetics.

Lazcano-Quintana, I., \& Caballo-Villar, B. (2016). Ocio deportivo juvenil: relación entre satisfacción e implicación organizativa. Revista de Psicología del Deporte, 25(2), 9-14.

López-Noguero, F., Sarrate Capdevila, M. L., \& Lebrero Baena, M. P. (2016). El ocio de los jóvenes en situación de vulnerabilidad. Análisis discursivo. Revista Española de Pedagogía, 263, 127-145.

Martínez, M. E. (2016). Deporte, integración y políticas sociales en contextos de vulneración. Madrid: Editorial Académica Española.

Melendro, M., García-Castilla, F. J., \& Goig Martínez, R. (2016). El uso de las TIC y la formación de los jóvenes vulnerables. Revista Española de Pedagogía, 263, 77-95

Ministerio de Educación, Cultura y Deporte (2015). Anuario de estadísticas deportivas. Madrid: Consejo Superior de Deportes. Recuperado de http://www.consejo-colef.es/descargas/Anuario_de_Estadisticas_Deportivas_2015.pdf

Ministerio de Sanidad, Servicios Sociales e Igualdad (2014). Encuesta Nacional de Salud. España 2011/12. Salud mental y calidad de vida en la población infantil. Serie Informes monográficos no 2. Madrid: Ministerio de Sanidad, Servicios Sociales e lgualdad. Recuperado de https://www.msssi.gob.es/estadEstudios/estadisticas/encuestaNacional/ encuestaNac2011/informesMonograficos/Act_fis_desc_ocio.4.pdf

OMS (2013). Physical activity promotion in socially disadvantaged groups: Principles for action. Denmark: WHO Regional Office for Europe. Recuperado de http://www.euro.who.int/_data/assets/pdf_file/o006/193092/PHAN-brochure_ENG.pdf?ua=1

Olive, L. S., Byrne, D. G., Cunningham, R. B., \& Telford, R. D. (2012). Effects of physical activity, fitness and fatness on children's body image: The Australian LOOK longitudinal study. Mental Health and Physical Activity, 5, 116-124. doi: https://doi.org/10.1016/ j.mhpa.2012.08.004

Pedisic, Z., Greblo, Z., Phongsavan, P., Milton, K., \& Bauman, A. E. (2015). Are total, intensity- and domain-specific physical activity levels associated with life satisfaction among university students? Plos One, 10(2). doi: https://doi. org/10.1371/journal. pone.0118137

Ramos, R., Ponce de León, A., \& Sanz, E. (2010). El ocio físico-deportivo en adolescentes. La Rioja: Universidad de La Rioja.

Ransdell, L. B., Vener, J. M., \& Sell, K. (2004). International perspectives: the influence of gender on lifetime physical activity participation. The journal of the Royal Society for the Promotion of Health, 124(1), 12-14. doi: https://doi. org/10.1177/146642400312400105

Sotomayor, P. L., Aquino, V. P., Jiménez, O. J., \& Trejo, M. C. (2014). Actividad física y sedentarismo: Determinantes sociodemográficos, familiares y su impacto en la salud del adolescente. Revista de Salúd Publica, 16(2), 161-172. doi: https://doi.org/10.15446/ rsap.v16n2.33329

Spaaij, R. (2009). Sport as a vehicle for social mobility and regulation of disadvantaged urban youth: Lessons from Rotterdam. International Review for the Sociology of Sport, 44 (2-3), 247-264. doi: https://doi.org/10.1177/1012690209338415

UNICEF (2010). Los niños dejados atrás. Una tabla clasificatoria de la desigualdad respecto al bienestar infantil en las naciones ricas del mundo. Florencia: UNICEF (Innocenti Research Centre).

Valdemoros, M. Á., Sanz, E., \& Ponce de León, A. (2012). Educación informal y ocio juvenil. El influjo de los amigos en el abandono de la práctica físico-deportiva. Pedagogía Social, 20, 203-221.

Valdemoros, M. Á., Sanz, E., \& Ponce de León, A. (2017). Ocio digital y ambiente familiar en estudiantes de Educación Postobligatoria. Comunicar: Revista científica iberoamericana de comunicación y educación, 50(5), 99-108.

Zullig, K. J., \& White, R. J. (2011). Physical activity, life satisfaction, and self-rated health of middle school students. Applied Research in Quality of Life, 6, 277-289. doi: https://doi.org/10.1007/s11482-010-9129-z

\section{Nota}

1 Aunque estas áreas son 10 (Noreste, Levante, Sur, Centro, Noroeste, Norte, Gran Canaria, Tenerife, Barcelona ciudad y Madrid ciudad), para facilitar el trabajo de campo y la eficiencia del modelo, se decidió reducir el número de áreas, integrando las zonas de Canarias en la zona Sur, Barcelona en la zona Noreste y Madrid en la zona Centro. 


\section{CÓMO CITAR ESTE ARTÍCULO}

Fraguela-Vale, R., De-Juanas, A., \& Franco. R. (2018). Ocio deportivo en jóvenes potencialmente vulnerables: beneficios percibidos y organización de la práctica. Pedagogía Social. Revista Interuniversitaria, 31, 49-58. DOI: 10.7179/PSRI_2018.31.04

\section{DIRECCIÓN COMPLETA DE LOS AUTORES}

Raúl Fraguela Vale. Universidad de A Coruña. Facultad de Ciencias de la Educación. Campus de Elviña, s/n. 15071 A Coruña. Telf.: 881014619. E-mail: raul.fraguela@udc.es

Ángel de-Juanas Oliva. Facultad de Educación UNED. Dpto. Teoría de la Educación y Pedagogía Social. C/Juan del Rosal 14. 28040. Madrid. Telf.: 9139869 79. E-mail: adejuanas@edu.uned.es

Ricardo Franco Lima: Escola Superior de Desporto e Lazer - IPVC. Complexo Desportivo e de Lazer Comendador Rui Solheiro, Monte de Prado, 4960-320 Melgaço - Portugal. www.esdl.ipvc. pt - E-mail: ricardo.lima@esdl.ipvc.pt

PERFIL ACADÉMICO

Raúl Fraguela Vale. Licenciado y Doctor en Educación Física por la Universidad de A Coruña. Titular de Escuela Universitaria en el área de Expresión Corporal de la Universidad de A Coruña. Sus principales líneas de investigación son: educación para el ocio y Educación Física, tiempos educativos y sociales, actividad física y salud en la infancia, políticas deportivas a nivel local y el juego en la infancia. Pertenece a los equipos de investigación SEPA (USC) y Política Educativa, Historia y Sociedad (UDC).

Ángel de-Juanas Oliva. Profesor Contratado Doctor en la Universidad Nacional de Educación a Distancia (UNED). Doctor en Ciencias de la Educación (UCM) y licenciado en Psicopedagogía. Coordinador del Master en Intervención Educativa en Contextos Sociales de la UNED. Miembro del grupo de investigación de Intervención Socioeducativa. Finalmente, es editor del Área de Enseñanza de la Educación Física de la revista RICYDE.

Ricardo Franco Lima. Doutorado em Ciências da Educação pela Universidade de Trás-os-Montes e Alto-Douro (2015). Professor de Ensino Superior desde 2010 no Instituto Universitário da Maia e no Instituto Politécnico de Viana do Castelo - Escola Superior de Educação e Escola Superior de Desporto e Lazer. Atualmente Coordenador da Licenciatura em Desporto e Lazer na Escola Superior de Desporto e Lazer de Melgaço, tendo como áreas de investigação a Pedagogia e Didática do Desporto e o Treino Desportivo (Voleibol). 